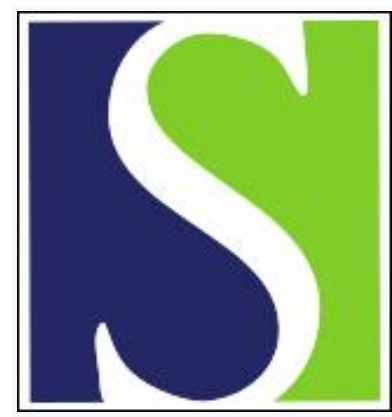

Scand J Work Environ Health 2012;38(5):393-408

https://doi.org/10.5271/sjweh.3275

Published online: 24 Jan 2012, Issue date: 01 Sep 2012

A systematic review of the cost-effectiveness of worksite physical activity and/or nutrition programs

by van Dongen JM, Proper KI, van Wier MF, van $\operatorname{der}$ Beek AJ, Bongers PM, van Mechelen W, van Tulder MW

Affiliation: Faculty of Earth and Life Sciences, Department of Health Sciences, Unit: Health Economics and Health Technology Assessment, Room: T-517, De Boelelaan 1085, 1081 HV Amsterdam, The Netherlands. j.m.van.dongen@vu.nl

Refers to the following texts of the Journal: 2004;30(1):36-46 2006;32(1):1-4 2007;33(3):161-164 2010;36(3):202-215 2010;36(4):273-288

The following articles refer to this text: 2013;39(3):217-220; 2018;44(5):458-474; 2020;46(2):127-142; 2022;48(4):249-252

Key terms: cost-effectiveness; dietary behavior; obesity; overweight; physical activity; review; systematic review; worksite health promotion; worksite physical activity

This article in PubMed: www.ncbi.nlm.nih.gov/pubmed/22270562

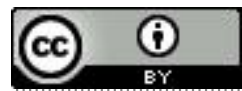




\title{
A systematic review of the cost-effectiveness of worksite physical activity and/or nutrition programs
}

\author{
by Johanna M van Dongen, MSc, 1, 2, 3 Karin I Proper, PhD, 1, 3 Marieke F van Wier, MSc, , 2, 4 Allard J van der \\ Beek, PhD, 1, 3 Paulien M Bongers, PhD, 1, 3, 5 Willem van Mechelen, PhD, 1, 3 Maurits W van Tulder, PhD 1, 2, 4
}

\begin{abstract}
van Dongen JM, Proper KI, van Wier MF, van der Beek AJ, Bongers PM, van Mechelen W, van Tulder MW. A systematic review of the cost-effectiveness of worksite physical activity and/or nutrition programs. Scand J Work Environ Health. 2012;38(5):393-408. doi:10.5271/sjweh.3275
\end{abstract}

Objective The aim of this study was to appraise and summarize the evidence on the cost-effectiveness of worksite physical activity and/or nutrition programs.

Methods We searched EMBASE, MEDLINE, SportDiscus, PsycInfo, NIOSHTIC-2, NHSEED, HTA, and Econlit for studies published up to 14 January 2011. Additionally, we searched for articles by reviewing references, searching authors' databases, and contacting authors of included studies. Two researchers independently selected articles. Articles had to include a cost-effectiveness and/or cost-utility analysis comparing a worksite physical activity and/or nutrition program to usual care or an abridged version of the program. Data were extracted on study characteristics and results. Two researchers independently assessed the risk of bias using the Consensus on Health Economic Criteria list (CHEC-list).

Results Ten studies (18 programs) were included. More than $50 \%$ of the studies fulfilled 11 (58\%) of the 19 CHEC-list items. From various perspectives, worksite nutrition and worksite physical activity and nutrition programs $(\mathrm{N}=6)$ were more costly and more effective in reducing body weight than usual care. When only intervention costs were considered, most worksite nutrition $(\mathrm{N}=4 / 5)$ and worksite physical activity and nutrition programs $(\mathrm{N}=5 / 6)$ were more costly and more effective in reducing cholesterol level and cardiovascular disease risks, respectively.

Conclusions The cost-effectiveness of more costly and more effective programs depends on the "willingness to pay" for their effects. It is unknown how much decision-makers are willing to pay for reductions in body weight, cholesterol level, and cardiovascular disease risks. Therefore, conclusions about the cost-effectiveness of worksite physical activity and/or nutrition programs cannot be made. There is substantial need for improvement of the methodological quality of studies and particular emphasis should be placed on the handling of uncertainty.

Key terms dietary behavior; obesity; overweight; worksite health promotion.

Regular physical activity and healthy dietary habits are considered important in preventing overweight, obesity, and their attributable diseases [eg, diabetes type 2, cardiovascular disease (CVD) and certain cancers] (1-5). Nevertheless, many adults do not meet public health recommendations for nutrition and physical activity (6-11). Currently, $34 \%$ of United States (US) adults are overweight [body mass index (BMI) $\geq 25 \mathrm{~kg} /$ $\mathrm{m}^{2}$ and $\left.<30 \mathrm{~kg} / \mathrm{m}^{2}\right]$ and $34 \%$ are obese (BMI $\geq 30 \mathrm{~kg} / \mathrm{m}$ ) (12). In Europe, the combined prevalence of overweight and obesity ranges from $38-61 \%$ among women and $52-69 \%$ among men (13). In addition to the toll that overweight and obesity take on the health and wellbeing of individuals, they impose considerable financial burdens in terms of increased productivity-related and healthcare costs (14-16). Therefore, health promotion

1 Body@Work, Research Center for Physical Activity, Work and Health, TNO-VU University Medical Center, Amsterdam, The Netherlands.

2 Dept Health Sciences and EMGO+ Institute for Health \& Care Research, Faculty of Earth \& Life Sciences, VU University Amsterdam, The Netherlands.

3 Dept Public \& Occupational Health and EMGO+ Institute for Health \& Care Research, VU University Medical Center, Amsterdam, The Netherlands.

4 Dept Epidemiology \& Biostatistics and EMGO+ Institute for Health \& Care Research, VU University Medical Center, Amsterdam, The Netherlands.

5 TNO Healthy Living, Hoofddorp, The Netherlands.

Correspondence to: Johanna M van Dongen, Faculty of Earth and Life Sciences, Department of Health Sciences, Unit: Health Economics and Health Technology Assessment, Room: T-517, De Boelelaan 1085, 1081 HV Amsterdam, The Netherlands. [E-mail: j.m.van.dongen@vu.nl] 
programs aimed at increasing physical activity and/or improving nutrition are warranted.

The worksite provides a useful setting for implementing such programs; since employees spend up to $60 \%$ of their waking hours at the worksite, organizational and social support can easily be made available, and large enterprises often have the infrastructure to offer such program at relatively low costs (17-19). Employers themselves may also benefit from implementing worksite health promotion (WHP) programs, as healthier workers are expected to be more productive and miss fewer days of work (17).

WHP programs aimed at increasing physical activity and/or improving nutrition were found effective in reducing body fat and body weight (20-22). For example, a recent systematic review found worksite physical activity and nutrition programs to significantly reduce body weight by $1.2 \mathrm{~kg}$, BMI by $0.3 \mathrm{~kg} / \mathrm{m}^{2}$, and body fat percentage by $1.1 \%$ during the first years after implementation ( $\leq 3$ years) (22). Budgets for occupational healthcare are restricted. Decisions about investments in WHP programs may, therefore, not only be guided by the evidence on their effectiveness, but also by considerations of their costs in relation to these effects (23-25). For this reason, cost-effectiveness analyses (CEA) and cost-utility analyses (CUA) are conducted to gain insight into the (additional) costs of an intervention per additional unit of effect gained. These analyses not only give insight into the cost savings of an intervention, like return on investment (ROI) analyses, but also provide details on the price of achieving a particular goal if an intervention produces better outcomes at additional costs (eg, costs per kilogram body weight loss) (21).

Although ROI results are likely to be most frequently used within companies to describe the financial aspects of a business case for occupational health initiatives $(26,27)$, CEA may be of interest for corporate decision-makers as well. A recent systematic review on the financial return of worksite physical activity and/or nutrition programs indicated that they may not pay for themselves in terms of reduced medical and/or absenteeism costs during the first years after implementation (28). Nevertheless, a significant lag between health improvements and reductions in medical and/or productivity-related cost may exist. Therefore, reporting on cost-effectiveness in terms of intermediate outcome measures that might be associated with long-term cost savings (eg, body weight loss) (29), may also give useful information to aid implementation decisions (17). Furthermore, investments in WHP programs may be motivated not only by making a profit but also by obtaining positive health effects and/or by the wish to be a caring employer. In that case, their anticipated effects are worth having and the question is to determine the most cost-effective way to achieve it (ie, least costly per unit of effect) (30).
Up until now, various reviews have been conducted on the cost-effectiveness of WHP programs (20, 31-39). One of them (36), for example, concluded that the literature provided "guarded cautious optimism" about their cost-effectiveness. However, these reviews were limited to studies published up until 2008 and most of them looked at the cost-effectiveness of WHP programs in general (ie, also including disease and stress management, and smoking cessation programs), instead of worksite physical activity and/or nutrition programs in particular. Furthermore, although the quality of the design and execution of economic evaluations should be considered when judging the validity of their findings, none of the reviews used an internationally accepted instrument for assessing their risk of bias $(40,41)$. This raises questions about the credibility of their conclusions. Therefore, the aim of the present study was critically to appraise and summarize the current evidence on the immediate and long-term cost-effectiveness of worksite physical activity and/or nutrition programs compared to usual care or an abridged version of the program.

\section{Methods}

\section{Search strategy}

A systematic search was conducted to identify studies evaluating the cost-effectiveness and/or cost-utility of WHP programs aimed at improving nutrition and/or increasing physical activity. Eight databases (EMBASE, MEDLINE, SPORTDiscus, PsycINFO, NIOSHTIC-2, NHSEED, HTA, and Econlit) were searched for studies published up to 14 January 2011. An information specialist of the VU University Medical Center was consulted to develop and run the search strategy. Databases were searched with the following keywords: participant/setting type (eg, "Workplace", "Employee", "Workforce"), intervention type (eg, "Health Promotion", "Lifestyle"), intervention aim (eg, "Exercise", "Physical Activity", "Nutrition", and "Diet"), and study design (eg, "Cost-Effectiveness Analysis", "Cost-Utility Analysis", and "Economic Evaluation"). In addition to the present study, a systematic review on the financial return of worksite physical activity and/or nutrition programs was conducted (28). Therefore, a broad search strategy was used so that the search results could be used for both studies simultaneously.

As an example, the complete search strategy for EMBASE can be found in the Appendix. In addition to the electronic search, reference lists of relevant review articles $(17,18,20,21,31-39,42)$ and those of the retrieved fulltext were searched. Articles were also identified from the authors' own literature databases. To 
identify unpublished studies, authors of included studies published during the last decade were contacted. During the search, a "search diary" was maintained, including keywords used, searched databases, and search results.

\section{Study selection}

Titles and abstracts of retrieved studies were stored in an electronic database using Reference Manager 11.0 (ISI Research Soft Inc, Berkeley, California). Two reviewers independently assessed whether these studies met the following inclusion criteria: (i) the study included a CEA and/or CUA, (ii) participants were part of the adult working population, (iii) the intervention under study was a WHP program aimed at improving nutrition and/ or increasing physical activity, (iv) the intervention was compared to usual care (including no intervention) or an abridged version of the program, (v) outcome measures included a behavioral measure (eg, physical activity and dietary intake), a health-related measure (eg, BMI, waist circumference, body fat percentage, musculoskeletal symptoms, cardiorespiratory fitness, and health risk profiles), or a work-related measure (eg, productivity and work satisfaction), and (vi) the study was reported in English, German, French, or Dutch.

For the purpose of this review, analyses could be performed from all perspectives (eg, employer's perspective and societal perspective). Furthermore, no limitations were set as to program format [eg, (self-) assessment, counseling, and exercise program], worksite characteristics (eg, age, gender, occupation, proportion of full-time employees, and number of employees), length of the intervention, and follow-up duration. Studies aimed at long-term sick-listed employees, employees with chronic conditions (eg, diabetes type 2 and CVD), retirees, and children were excluded. If studies met the inclusion criteria, or if uncertainty remained about inclusion, fulltexts were retrieved. All fulltexts were read and checked for eligibility. To resolve disagreements between the two reviewers, a consensus procedure was used. A third reviewer was consulted when disagreements persisted; this was necessary on one occasion.

\section{Risk of bias assessment}

Two reviewers independently assessed the risk of bias of included studies. If one of the reviewers was a (co-)author of a given study, another reviewer acted as the second reviewer. Risk of bias was assessed using the Consensus Health Economic Criteria list (CHEC-list), which was developed for systematic reviews of economic evaluations using a Delphi consensus procedure involving 23 international experts in economic evaluations (43). The test-retest reliability of the CHEC-list was shown to be good (intra-class correlation coefficient: $0.97,95 \%$ CI $0.73-0.98)$ (44). Items were scored as negative in case of an inadequate performance of an item or if insufficient information was available in the article or related materials (43). If a study presented its results in multiple articles, those articles were scored as one study. A consensus procedure was used to resolve disagreements between the two reviewers. When disagreements remained, a third reviewer was consulted; this was necessary on two occasions.

Data extraction, data analyses, and applied classification schemes

Data were extracted on: (i) study details (eg, perspective, primary study design, setting, and follow-up duration), (ii) characteristics of the study population (eg, participant and job characteristics), (iii) program focus (ie, improving nutrition, increasing physical activity, or both), (iv) program format [ie, (self-)assessment, educational/informational, behavioral, exercise, environmental, and incentive components], (v) measurement and valuation methods of costs, (vi) measurement methods of effects, and (vii) study results [reported costs, effects, and incremental cost-effectiveness ratios (ICER)]. One reviewer extracted data using a pre-designed data extraction form. Ten percent of the extracted data was checked by a second reviewer, which did not reveal any errors. If articles did not contain sufficient information on study results, authors were contacted for missing data.

If an incremental cost-effectiveness analysis was not performed, an ICER was calculated per reported outcome measure as the incremental difference in costs relative that in effects (30). Costs and ICER were standardized to 2010 US dollars (USD) using consumer price indices (45) and purchasing power parities (46). For this, their reference year was needed. If their reference year was not stated, the year of publication was used. For data analyses and presentation, studies were grouped according to their program focus (ie, improving nutrition, increasing physical activity, or both), (stated) perspective, and outcome measures.

To summarize results, and thereby draw conclusions about the cost-effectiveness of the included programs, their incremental costs and effects were explored. Programs that were less costly and more effective than the control condition were considered cost-effective (ie, the program dominates the control condition). For programs that were more costly and less effective, the opposite was true. Programs that were either more costly and more effective or less costly and less effective were only considered cost-effective if their ICER was respectively lower or higher than the "willingness to pay" (ie, the maximum amount of money decision-makers are willing to pay per unit of effect gained) (30). 


\section{Results}

\section{Literature search and study selection}

The electronic search yielded 3230 unique references. After screening their abstracts and titles, we retrieved 47 fulltexts. Thirty-one additional fulltexts were retrieved after screening references of relevant review articles and those of the retrieved fulltexts. After reading those 78 fulltexts, 9 articles were identified that met the inclusion criteria (figure 1). Additionally, three unpublished articles were identified by searching the authors' own literature databases. Contacting authors of included studies did not yield any additional results. Most studies were excluded because they did not include an economic evaluation or because they only evaluated the financial return by comparing intervention costs to their financial consequences. Eventually, 12 articles, including 10 original studies (47-58), were included in the review.

\section{Study characteristics}

A description of the study characteristics can be found in table 1 . Worksite nutrition programs $(\mathrm{N}=7)$ were evaluated in four studies $(47,51,55,56)$ and worksite physical activity and nutrition programs $(\mathrm{N}=11)$ in six studies $(48-50,52-54,57,58)$. None of the studies evaluated a WHP program solely aimed at increasing physical activity. In general, interventions consisted of a (self-)assessment, educational/informational, behavioral, exercise, environmental, and/or incentive component. All interventions were compared to usual care, consisting of no intervention or a (self-) assessment, educational/informational, and/or environmental component. The number of participants in the studies ranged from 66-1883. The length of the interventions ranged from 12 weeks to 3 years. Four studies $(51,54-56)$ evaluated the short-term effectiveness of the programs (follow-up $\leq 6$ months) and six studies $(47-50,52,53,57,58)$ evaluated the long-term

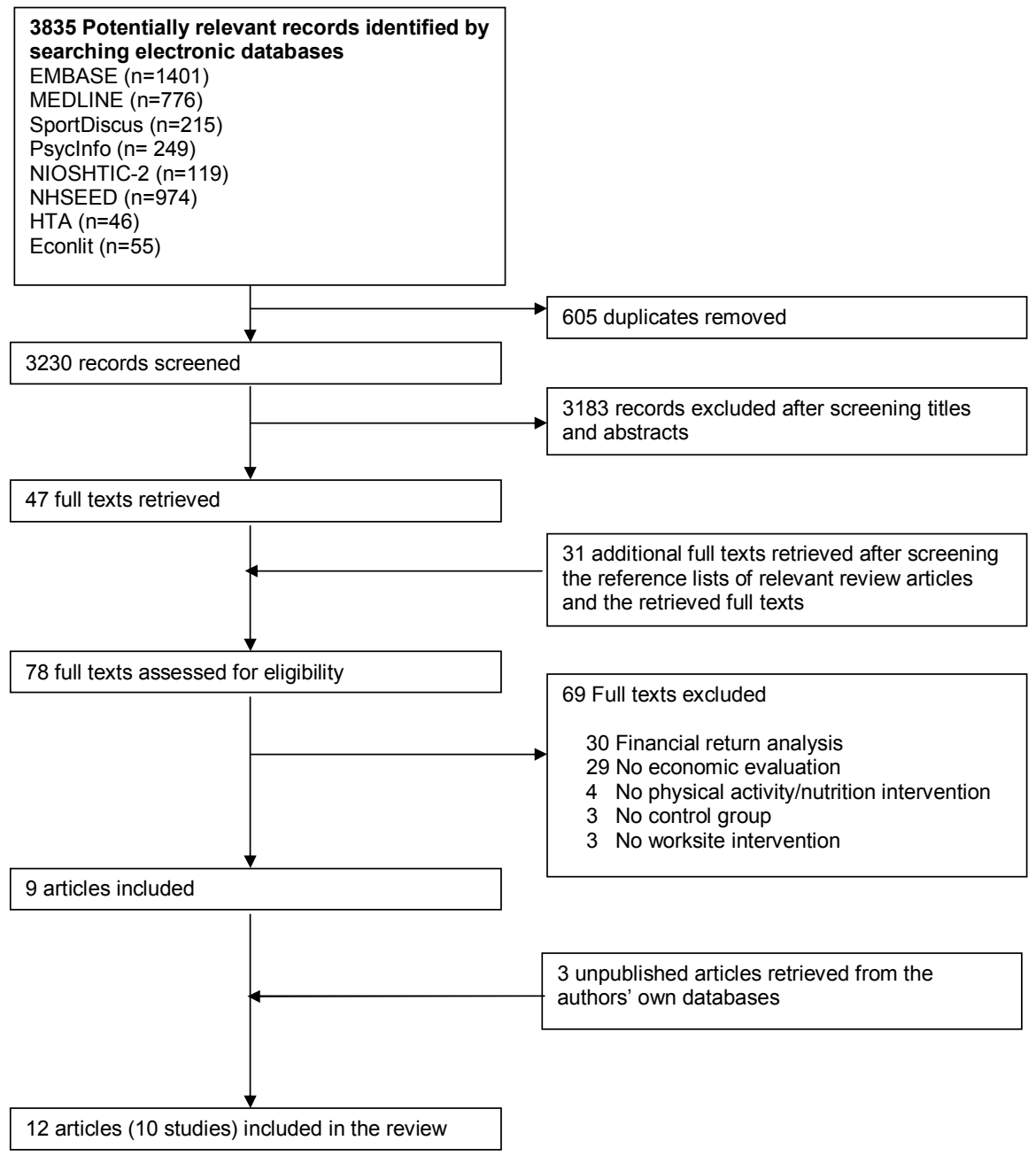


Table 1. Characteristics of the included studies $(\mathrm{N}=10)$. [ $\mathrm{BC}=$ behavioral counseling; $\mathrm{BCl}=$ behavioral counseling plus incentives; CVD=Cardiovascular disease; FC=fitness centre; HEC=health education \& follow-up counseling; HECE=health education, follow-up counseling \& environmental strategies; HRA=Health risk assessment; I=Intervention group; NL=The Netherlands; NRS=Non-randomized trial; NS=Not stated; PA=Physical activity; RCT=Randomized controlled trial; RFE=risk factor education; SF-36=Short Form-36; UC=Usual care; WEL=Weight efficacy lifestyle; WPAI=Work productivity and activity impairment]

\begin{tabular}{|c|c|c|c|c|c|c|}
\hline Study & Study details & Population & $\begin{array}{l}\text { Intervention and control } \\
\text { conditions }\end{array}$ & $\begin{array}{l}\text { Intervention } \\
\text { purpose, format }\end{array}$ & Costs & Outcomes \\
\hline \multicolumn{7}{|c|}{ Worksite nutrition program } \\
\hline $\begin{array}{l}\text { Katcher } \\
\text { et al (51) }\end{array}$ & $\begin{array}{l}\text { Perspective: NS } \\
\text { Primary study: NRS } \\
\text { Setting: USA 2007- } \\
\text { 2008; } \\
\text { Length intervention: } \\
22 \text { weeks; follow- } \\
\text { up: } 22 \text { weeks }\end{array}$ & $\begin{array}{l}113 \text { overweight } \\
\text { employees and/ } \\
\text { or employees } \\
\text { with an elevated } \\
\text { type } 2 \text { diabetes } \\
\text { risk from insur- } \\
\text { ance company } \\
\text { UC: } 45 ; \text {;: } 68\end{array}$ & $\begin{array}{l}\text { UC: - } \\
\text { I: Weekly (vegan) diet } \\
\text { instructions ( } 22 \text { ses- } \\
\text { sions), daily low-fat vegan } \\
\text { options in cafeteria, daily } \\
\text { multivitamin }\end{array}$ & $\begin{array}{l}\text { Purpose: improving } \\
\text { health-related } \\
\text { quality of life and } \\
\text { work productivity } \\
\text { Format: UC: - } \\
\text { | a, b }\end{array}$ & $\begin{array}{l}\text { Intervention costs: } \\
\text { micro-costed, } \\
\text { valuation method } \\
\text { NS }\end{array}$ & $\begin{array}{l}\text { Body weight: digital } \\
\text { scale, light clothing, } \\
\text { without shoes ( } 70) ; \\
\text { Eating behavior: The } \\
\text { Eating Inventory; Health- } \\
\text { related quality of life: SF- } \\
\text { 36; Work productivity: } \\
\text { WPAI questionnaire }\end{array}$ \\
\hline $\begin{array}{l}\text { Siggaard } \\
\text { et al (55) }\end{array}$ & $\begin{array}{l}\text { Perspective: NS } \\
\text { Primary study: NRS } \\
\text { Setting: Denmark } \\
\text { (year NS) } \\
\text { Length intervention: } \\
12 \text { weeks; follow- } \\
\text { up: } 12 \text { weeks }\end{array}$ & $\begin{array}{l}66 \text { insurance } \\
\text { company } \\
\text { employees } \\
\text { UC: } 16 \\
\text { I: } 50\end{array}$ & $\begin{array}{l}\text { UC: - } \\
\text { I: Nutrition education } \\
\text { (12 sessions), weekly } \\
\text { weightings }\end{array}$ & $\begin{array}{l}\text { Purpose: weight } \\
\text { loss } \\
\text { Format: UC: - } \\
\text { | a,c }\end{array}$ & $\begin{array}{l}\text { Intervention costs: } \\
\text { micro-costed, } \\
\text { valuation method } \\
\text { NS }\end{array}$ & $\begin{array}{l}\text { Body weight: digital } \\
\text { scale, light clothing, } \\
\text { sober for } 3 \text { hours; Daily } \\
\text { carbohydrate and fat in- } \\
\text { take: 4-day dietary record }\end{array}$ \\
\hline $\begin{array}{l}\text { Wilson } \\
\text { et al (56) }\end{array}$ & $\begin{array}{l}\text { Perspective: NS } \\
\text { Primary study: NRS } \\
\text { Setting: USA (year } \\
\text { NS) } \\
\text { Length intervention: } \\
\text { 1-3 months; follow- } \\
\text { up: } 6 \text { months }\end{array}$ & $\begin{array}{l}652 \text { manufac- } \\
\text { turing workers } \\
\text { with an elevated } \\
\text { CVD risk } \\
\text { UC: } 146 \\
\text { I-1: } 180 \\
\text { I-2: } 161 \\
\text { I-3: } 112 \\
\text { I-4: } 53\end{array}$ & $\begin{array}{l}\text { UC: Cholesterol screening, } \\
\text { health education materials } \\
\text { I-1: Cholesterol screening, } \\
\text { health education materi- } \\
\text { als, 1-month educational } \\
\text { program } \\
\text { I-2: Cholesterol screening, } \\
\text { health education materials, } \\
\text { 1-month educational pro- } \\
\text { gram, incentives } \\
\text { I-3: Cholesterol screening, } \\
\text { health education materi- } \\
\text { als, 3-month educational } \\
\text { program } \\
\text { I-4: Cholesterol screening, } \\
\text { health education materials, } \\
\text { 3-month educational pro- } \\
\text { gram, incentives }\end{array}$ & $\begin{array}{l}\text { Purpose: CVD risk } \\
\text { reduction } \\
\text { Format: UC: a, c } \\
\text { I-1: a, c } \\
\text { I-2: a, c, } \\
\text { I-3: } a, c \\
\text { I-4: } a, c, d\end{array}$ & $\begin{array}{l}\text { Intervention costs: } \\
\text { micro-costed, val- } \\
\text { ued using } \\
\text { tariffs and depleted } \\
\text { sources }\end{array}$ & $\begin{array}{l}\text { Cholesterol level reduc- } \\
\text { tion: percentage of par- } \\
\text { ticipants with } 10 \% \text { (or } \\
\text { more) cholesterol level } \\
\text { reduction, measured } \\
\text { using the Reflotrol dry } \\
\text { chemical analyzer }\end{array}$ \\
\hline $\begin{array}{l}\text { Byers } \\
\text { et al (47) }\end{array}$ & $\begin{array}{l}\text { Perspective: NS } \\
\text { Primary study: RCT } \\
\text { Setting: USA (year } \\
\text { NS) } \\
\text { Length intervention: } \\
1 \text { month; follow-up: } \\
12 \text { months }\end{array}$ & $\begin{array}{l}846 \text { employees } \\
\text { with an elevated } \\
\text { CVD risk (vari- } \\
\text { able companies) } \\
\text { UC: } 463 \\
\text { I: } 383\end{array}$ & $\begin{array}{l}\text { UC: Cholesterol screening, } \\
\text { health education materials } \\
\text { I: Cholesterol screening, } \\
\text { health education materials, } \\
\text { nutrition education ses- } \\
\text { sions ( } 2 \text { hours), } 30 \text { minute } \\
\text { videocassette on nutrition }\end{array}$ & $\begin{array}{l}\text { Purpose: CVD risk } \\
\text { reduction } \\
\text { Format: UC: a, c } \\
\text { I: a, c }\end{array}$ & $\begin{array}{l}\text { Intervention costs: } \\
\text { micro-costed, val- } \\
\text { ued using } \\
\text { tariffs and depleted } \\
\text { sources }\end{array}$ & $\begin{array}{l}\text { Cholesterol level } \\
\text { reduction: percentage } \\
\text { cholesterol level r } \\
\text { eduction, measured } \\
\text { using the Reflotrol dry } \\
\text { chemical analyzer or the } \\
\text { Kodak system }\end{array}$ \\
\hline \multicolumn{7}{|c|}{$\begin{array}{l}\text { Worksite physical activity and } \\
\text { nutrition programs }\end{array}$} \\
\hline $\begin{array}{l}\text { Rasu et al } \\
(54)\end{array}$ & $\begin{array}{l}\text { Perspective: imple- } \\
\text { menting agency } \\
\text { Primary study: RCT } \\
\text { Setting: USA 2003- } \\
2006 \\
\text { Length intervention: } \\
6 \text { months; follow- } \\
\text { up: } 6 \text { months }\end{array}$ & $\begin{array}{l}442 \text { overweight } \\
\text { US Air Force } \\
\text { employees } \\
\text { UC: } 215 \\
\text { I: } 227\end{array}$ & $\begin{array}{l}\text { UC: HRA, standard face-to- } \\
\text { face nutrition/PA programs } \\
\text { I: HRA, standard face- } \\
\text { to-face nutrition/PA pro- } \\
\text { grams, in person treatment } \\
\text { orientation meeting \& } 24 \\
\text { week behavioral internet } \\
\text { therapy, manual for weight } \\
\text { control, telephone counsel- } \\
\text { ing ( } 2 \text { times) }\end{array}$ & $\begin{array}{l}\text { Purpose: weight } \\
\text { loss } \\
\text { Format: UC: a, c } \\
\text { I: }{ }^{a, c, e}\end{array}$ & $\begin{array}{l}\text { Intervention costs: } \\
\text { micro-costed, } \\
\text { valued using tar- } \\
\text { iffs and depleted } \\
\text { sources }\end{array}$ & $\begin{array}{l}\text { Body weight: Calibrated } \\
\text { scale, indoor clothes, } \\
\text { without shoes; } \\
\text { Waist circumference: } \\
\text { Gulick tape at the um- } \\
\text { bilicus; } \\
\text { Weight efficacy lifestyle: } \\
\text { WEL questionnaire }\end{array}$ \\
\hline $\begin{array}{l}\text { Groeneveld } \\
\text { et al (50) }\end{array}$ & $\begin{array}{l}\text { Perspective: societal } \\
\text { Primary study: RCT } \\
\text { Setting: NL 2007- } \\
2009 \\
\text { Length intervention: } \\
6 \text { months; follow- } \\
\text { up: } 12 \text { months }\end{array}$ & $\begin{array}{l}573 \text { construc- } \\
\text { tion workers } \\
\text { with an elevated } \\
\text { CVD risk } \\
\text { UC: } 280 \\
\text { I: } 293\end{array}$ & $\begin{array}{l}\text { UC: HRA, health education } \\
\text { materials } \\
\text { I: HRA, health education } \\
\text { materials, } 6 \text {-month coun- } \\
\text { seling program ( } 3 \text { face-to- } \\
\text { face sessions / } 4 \text { telephone } \\
\text { contacts) }\end{array}$ & $\begin{array}{l}\text { Purpose: CVD risk } \\
\text { reduction } \\
\text { Format } \\
\text { UC: } a, c \\
\text { I: } a, c, e\end{array}$ & $\begin{array}{l}\text { Intervention costs: } \\
\text { micro-costed, } \\
\text { valued using tariffs } \\
\text { and depleted sourc- } \\
\text { es; Absenteeism } \\
\text { costs: self-reported } \\
\text { days missed due to } \\
\text { health complaints, } \\
\text { multiplied by an } \\
\text { average wage rate; } \\
\text { Medical costs: Self- } \\
\text { reported healthcare } \\
\text { utilization valued } \\
\text { using Dutch } \\
\text { standard costs }\end{array}$ & $\begin{array}{l}\text { Body weight: Digital } \\
\text { scale, no shoes, no } \\
\text { jacket }\end{array}$ \\
\hline
\end{tabular}


Table 1. Continued

\begin{tabular}{|c|c|c|c|c|c|c|}
\hline Study & Study details & Population & $\begin{array}{l}\text { Intervention and control } \\
\text { conditions }\end{array}$ & $\begin{array}{l}\text { Intervention } \\
\text { purpose, format }\end{array}$ & Costs & Outcomes \\
\hline $\begin{array}{l}\text { van wier } \\
\text { et al } \\
(58)\end{array}$ & $\begin{array}{l}\text { Perspective: societal } \\
\text { Primary study: RCT } \\
\text { Setting: NL 2004- } \\
2006 \\
\text { Length intervention: } \\
6 \text { months; follow- } \\
\text { up: } 24 \text { months }\end{array}$ & $\begin{array}{l}1386 \text { over- } \\
\text { weight employ- } \\
\text { ees (variable } \\
\text { companies) } \\
\text { UC: } 460 \\
\text { I-Phone: } 462 \\
\text { I-Internet: } 464\end{array}$ & $\begin{array}{l}\text { UC: health education mate- } \\
\text { rials \& oral instructions } \\
\text { I- Phone: ring binder con- } \\
\text { taining } 10 \text { health education } \\
\text { modules, telephone coun- } \\
\text { seling, pedometer, health } \\
\text { education materials \& oral } \\
\text { instructions; I-Internet: } \\
\text { website containing } 10 \\
\text { health education modules, } \\
\text { email counseling, pedom- } \\
\text { eter, health education ma- } \\
\text { terials \& oral instructions }\end{array}$ & $\begin{array}{l}\text { Purpose: weight } \\
\text { Ioss } \\
\text { Format: UC: a } \\
\text { I-Phone: }{ }^{a, e} \\
\text { I-Internet: a, e }\end{array}$ & $\begin{array}{l}\text { Intervention costs: } \\
\text { micro-costed, val- } \\
\text { ued using tariffs } \\
\text { and depleted sourc- } \\
\text { es; Absenteeism } \\
\text { costs: Self-reported } \\
\text { number of days } \\
\text { missed due to } \\
\text { health complaints } \\
\text { multiplied by an } \\
\text { average wage rate; } \\
\text { Medical costs: Self- } \\
\text { reported healthcare } \\
\text { utilization valued } \\
\text { using Dutch stan- } \\
\text { dard costs }\end{array}$ & $\begin{array}{l}\text { Body weight: digital } \\
\text { scale, light clothes, no } \\
\text { shoes. When digital } \\
\text { weight measurements } \\
\text { were missing, self re- } \\
\text { ported weight measure- } \\
\text { ments were used; } \\
\text { Quality-adjusted life } \\
\text { years: EuroQol scores } \\
\text { valued using Dutch tar- } \\
\text { iffs and multiplied with } \\
\text { the amount of time spent } \\
\text { in a specific health state }\end{array}$ \\
\hline $\begin{array}{l}\text { Gussen- } \\
\text { hoven et al } \\
\text { (57) }\end{array}$ & $\begin{array}{l}\text { Perspective: } \\
\text { employer } \\
\text { Primary study: RCT } \\
\text { Setting: NL 2004- } \\
2005 \\
\text { Length intervention: } \\
6 \text { months; follow- } \\
\text { up: } 12 \text { months }\end{array}$ & $\begin{array}{l}\text { See van wier et } \\
\text { al }(58)\end{array}$ & See van wier et al (58) & $\begin{array}{l}\text { See van wier } \\
\text { et al (58) }\end{array}$ & $\begin{array}{l}\text { Intervention } \\
\text { costs: micro- } \\
\text { costed, valued } \\
\text { using tariffs and } \\
\text { depleted sources; } \\
\text { Absenteeism } \\
\text { costs: number of } \\
\text { days missed due to } \\
\text { health complaints } \\
\text { based on disability } \\
\text { absence data mul- } \\
\text { tiplied by an aver- } \\
\text { age wage rate }\end{array}$ & $\begin{array}{l}\text { Body weight: self-report- } \\
\text { ed body weight. When } \\
\text { self reported body weight } \\
\text { data was missing, digital } \\
\text { weight measurements } \\
\text { were used }\end{array}$ \\
\hline $\begin{array}{l}\text { Oldenburg } \\
\text { et al (52) }\end{array}$ & $\begin{array}{l}\text { Perspective: NS } \\
\text { Primary study: RCT } \\
\text { Setting: Australia } \\
\text { (year NS) } \\
\text { Length intervention: } \\
12 \text { months; follow- } \\
\text { up: } 12 \text { months }\end{array}$ & $\begin{array}{l}431 \text { ambulance } \\
\text { workers } \\
\text { UC: } 130 \\
\text { I-RFE: } 82 \\
\text { I-BC: } 124 \\
\text { I-BCI: } 95\end{array}$ & $\begin{array}{l}\text { UC: HRA } \\
\text { I-RFE: HRA, standardized } \\
\text { healthy lifestyle advice, ed- } \\
\text { ucational resource manual } \\
\text { and videotape; } \\
\text { I-BC: HRA, standardized } \\
\text { healthy lifestyle advice, ed- } \\
\text { ucational resource manual } \\
\text { and videotape, counseling } \\
\text { sessions for participants } \\
\text { with a high CVD risk, self } \\
\text { instruction lifestyle change } \\
\text { manual } \\
\text { I-BCI: HRA, standardized } \\
\text { healthy lifestyle advice, ed- } \\
\text { ucational resource manual } \\
\text { and videotape, counseling } \\
\text { sessions for participants } \\
\text { with a high CVD risk, self } \\
\text { instruction lifestyle change } \\
\text { manual, incentives }\end{array}$ & $\begin{array}{l}\text { Purpose: CVD risk } \\
\text { reduction } \\
\text { Format: UC: c } \\
\text { I-RFE: a, c } \\
\text { I-BC: a, c, e } \\
\text { I-BCI: a, c, e, d }\end{array}$ & $\begin{array}{l}\text { Intervention costs: } \\
\text { micro-costed, } \\
\text { valued using } \\
\text { tariffs and depleted } \\
\text { sources }\end{array}$ & $\begin{array}{l}\text { Changes in CVD risk: } \\
\text { composite score, includ- } \\
\text { ing; cholesterol level, } \\
\text { blood pressure, number } \\
\text { cigarettes smoked, body } \\
\text { mass index, maximum } \\
\text { oxygen uptake }\end{array}$ \\
\hline $\begin{array}{l}\text { Erfurt et al } \\
(48)\end{array}$ & $\begin{array}{l}\text { Perspective: NS } \\
\text { Primary study: RCT } \\
\text { Setting: USA 1985- } \\
1988 \\
\text { Length intervention: } \\
3 \text { years; follow-up: } \\
3 \text { years }\end{array}$ & $\begin{array}{l}1607 \text { manufac- } \\
\text { turing workers } \\
\text { with an elevated } \\
\text { CVD risk ( } \geq 1 \text { of } \\
3 \text { CVD risks) } \\
\text { UC: } 420 \\
\text { I-HE: } 432 \\
\text { I-HEC: } 408 \\
\text { I-HECE: } 347\end{array}$ & $\begin{array}{l}\text { UC: HRA, health improve- } \\
\text { ment classes, fitness centre } \\
\text { I-HE: HRA, health improve- } \\
\text { ment classes, wellness } \\
\text { committee, health education } \\
\text { strategies } \\
\text { I-HEC: HRA, health improve- } \\
\text { ment classes, wellness } \\
\text { committee, health educa- } \\
\text { tion strategies, counsel- } \\
\text { ing \& health improvement } \\
\text { programs for high risk } \\
\text { employees. } \\
\text { I-HECE: HRA, health im- } \\
\text { provement classes, wellness } \\
\text { committee, health educa- } \\
\text { tion strategies, counsel- } \\
\text { ing \& health improvement } \\
\text { programs for high risk em- } \\
\text { ployees, health communica- } \\
\text { tion networks, peer support } \\
\text { groups, health promotion } \\
\text { groups, organizational strat- } \\
\text { egies to encourage/support } \\
\text { health improvements }\end{array}$ & $\begin{array}{l}\text { Purpose: CVD risk } \\
\text { reduction Format: } \\
\text { UC: a, c, f } \\
\text { I-HE: a, b, c } \\
\text { I-HEC: a, b, e, f } \\
\text { I-HECE: a, b, c, e, f }\end{array}$ & $\begin{array}{l}\text { Intervention costs: } \\
\text { Micro-costed, } \\
\text { valued using tar- } \\
\text { iffs and depleted } \\
\text { sources }\end{array}$ & $\begin{array}{l}\text { Changes in CVD risk: } \\
\text { Percentage of three } \\
\text { risk factors (high blood } \\
\text { pressure, overweight } \\
\text { and smoking) that were } \\
\text { moderately and highly } \\
\text { reduced or prevented }\end{array}$ \\
\hline
\end{tabular}


Table 1. Continued

\begin{tabular}{|c|c|c|c|c|c|c|}
\hline Study & Study details & Population & $\begin{array}{l}\text { Intervention and control } \\
\text { conditions }\end{array}$ & $\begin{array}{l}\text { Intervention } \\
\text { purpose, format }\end{array}$ & Costs & Outcomes \\
\hline $\begin{array}{l}\text { Erfurt et al } \\
(49)\end{array}$ & See Erfurt et al (48) & $\begin{array}{l}1883 \text { manufac- } \\
\text { turing workers } \\
\text { with an elevated } \\
\text { CVD risk }(\geq 1 \text { of } \\
4 \text { CVD risks) } \\
\text { UC: } 493 \\
\text { I-FC: } 505 \\
\text { I-HEC: } 482 \\
\text { I-HECE: } 403\end{array}$ & $\begin{array}{l}\text { UC: I-HE Erfurt et al (48) } \\
\text { I-FC: "UC" Erfurt et al (48) } \\
\text { I-HEC: "I-HEC" Erfurt et } \\
\text { al (48) } \\
\text { I-HECE: "I-HECE" Erfurt } \\
\text { et al (48) }\end{array}$ & $\begin{array}{l}\text { Purpose: CVD risk } \\
\text { reduction } \\
\text { Format: UC: a, b, c } \\
\text { I-FC: a, c, }, \text { } \\
\text { I-HEC: } a, b, c, e \\
\text { I-HECE: } a, b, c, e, f\end{array}$ & $\begin{array}{l}\text { See Erfurt et al } \\
\text { (48) }\end{array}$ & $\begin{array}{l}\text { Changes in CVD risk: } \\
\text { percentage of four risk } \\
\text { factors (high blood pres- } \\
\text { sure, overweight, smok- } \\
\text { ing and lack of exercise) } \\
\text { that were moderately } \\
\text { and highly reduced or } \\
\text { prevented }\end{array}$ \\
\hline $\begin{array}{l}\text { Proper } \\
\text { et al (53) }\end{array}$ & $\begin{array}{l}\text { Perspective: } \\
\text { employer } \\
\text { Primary study: RCT } \\
\text { Setting: NL 2000- } \\
2002 \\
\text { Length intervention: } \\
9 \text { months; follow- } \\
\text { up: } 9 \text { months }\end{array}$ & $\begin{array}{l}299 \text { civil } \\
\text { servants } \\
\text { UC: } 168 \\
\text { I: } 131\end{array}$ & $\begin{array}{l}\text { UC: Health education } \\
\text { materials } \\
\text { I: Health education mate- } \\
\text { rial, 9-month counseling } \\
\text { program (7 face-to-face } \\
\text { sessions) }\end{array}$ & $\begin{array}{l}\text { Purpose: improving } \\
\text { physical fitness } \\
\text { Format: UC: }{ }^{a} \\
\text { I: a, e }\end{array}$ & $\begin{array}{l}\text { Intervention costs: } \\
\text { micro-costed, val- } \\
\text { ued using } \\
\text { tariffs and de- } \\
\text { pleted sources; } \\
\text { Absenteeism } \\
\text { costs: number of } \\
\text { days missed due } \\
\text { to health condition } \\
\text { based on disability } \\
\text { absence data mul- } \\
\text { tiplied by an aver- } \\
\text { age wage rate }\end{array}$ & $\begin{array}{l}\text { Meeting PA recommen- } \\
\text { dations: self-reported; } \\
\text { Energy expenditure: } \\
\text { structured interview (7- } \\
\text { day PA recall); } \\
\text { Sub-max heart rate: } \\
\text { bicycle ergometer test; } \\
\text { Upper-extremity } \\
\text { symptoms: Nordic } \\
\text { questionnaire }\end{array}$ \\
\hline
\end{tabular}

a Education/Information program format.

${ }^{b}$ Environment program format.

c Self-assessment program format.

d Incentives program format.

${ }^{\text {e }}$ Behavioral program format.

${ }^{\dagger}$ Exercise program format.

effectiveness (follow-up $>6$ months). No studies had a follow-up $>3$ years. Seven studies $(47-50,52-54,57$, 58 ) were conducted alongside a randomized controlled trial and three $(51,55,56)$ alongside a non-randomized study. Five studies $(47-49,51,54,56)$ were conducted in the US, three $(50,53,57,58)$ in the Netherlands, one (52) in Australia, and one (55) in Denmark. All studies conducted a CEA, and one (58) also conducted a CUA.

\section{Risk of bias assessment}

Reviewers initially disagreed on $40(21 \%)$ of the 190 items (10 studies multiplied with 19 CHEC-list items). Most disagreements were due to reading errors and different interpretations of the CHEC-list items and were solved during the consensus meeting; for four disagreements a third reviewer was consulted. Of the 19 CHEC-list items, 11 (58\%) were fulfilled by more than $50 \%$ of the studies and 7 items $(37 \%)$ by more than $75 \%$. The economic perspective was specifically stated by four studies $(50,53,54,57,58)$, including: the societal perspective, the employer's perspective, and that of an implementing agency. The latter (54) appropriately collected costs to the chosen perspective by only including intervention costs. Studies performed from the societal and employer's perspective also included absenteeism and/or medical costs and were all conducted in The Netherlands.
Costs were measured in physical units [ie, individual items of an intervention were measured (30)] in four studies, $(52-54,57,58)$. One of them (54) also appropriately valued costs by calculating them based on depleted sources [ie, based on the value of the forgone benefits because the resources were not available for their best alternative use (30)] and stating their reference year. Three studies $(47,51,56)$ presented both costs and effects, but did not conduct an incremental cost-effectiveness analysis. Just over half of the studies conducted a sensitivity analysis to test the robustness of their results (table 2).

\section{Cost effectiveness analysis}

Worksite nutrition programs. All four studies (47, 51, $55,56)$ evaluating WHP programs aimed at improving nutrition only included intervention costs in their cost estimates (table 3$)$. Two of them $(51,55)$ evaluated cost-effectiveness by comparing intervention costs to the effect on body weight reduction. Both interventions were more costly and more effective than usual care at a cost of $\$ 43$ and $\$ 20$ per kilogram body weight loss (see also table 4). One of those (55) was also more costly and more effective in reducing daily fat intake and increasing daily carbohydrate intake. The other intervention (51) was also more costly and more effective in improving physical functioning, general health, 
Table 2. Risk of bias assessment of included studies using the Consensus on Health Economic Criteria list (CHEC-list).

\begin{tabular}{|c|c|c|}
\hline \multirow[t]{2}{*}{ CHEC-list items } & \multicolumn{2}{|c|}{$\begin{array}{l}\text { Studies scoring } \\
\qquad N=10\end{array}$} \\
\hline & $\mathrm{N}$ (Yes) & $\%$ \\
\hline 1) Study population & 7 & 70 \\
\hline 2) Competing alternatives & 9 & 90 \\
\hline 3) Research question & 5 & 50 \\
\hline 4) Study design & 10 & 100 \\
\hline 5) Time horizon & 9 & 90 \\
\hline 6) Perspective & 3 & 30 \\
\hline 7) Costs identified & 1 & 10 \\
\hline 8) Costs measured & 4 & 40 \\
\hline 9) Costs valued & 1 & 10 \\
\hline 10) Outcomes identified & 9 & 90 \\
\hline 11) Outcomes measured & 8 & 80 \\
\hline 12) Outcomes valued & 10 & 100 \\
\hline 13) Incremental analysis & 7 & 70 \\
\hline 14) Discounted & 7 & 70 \\
\hline 15) Sensitivity analysis & 6 & 60 \\
\hline 16) Conclusions & 10 & 100 \\
\hline 17) Generalizability & 1 & 10 \\
\hline 18) Conflict of interest & 2 & 20 \\
\hline 19) Ethical and distributional issues & 0 & 0 \\
\hline
\end{tabular}

vitality, mental health, impairment at work, and impairment with daily activities. However, the intervention was more costly and less effective in reducing restraint, disinhibition (ie, overeating in response to stress or other cues), and hunger.

Two other studies $(47,56)$ evaluated cost-effectiveness by comparing intervention costs to the effect on cholesterol level reduction. However, both studies used different outcomes for assessing the degree of cholesterol level reduction, which limits their comparability. The first study (56) evaluated four different interventions (I): (i) I-1: 1-month program without incentives, (ii) I-2: 1-month program with incentives, (iii) I-3: 3 -month program without incentives, and (iv) I-4: 3 -month program with incentives. The least intensive program (ie, 1-month program without incentives) was more costly and less effective than usual care (ICER: \$-110 per $1 \%$ of participants reducing their cholesterol level by $\geq 10 \%$ ). The other interventions were more costly and more effective (ICER I-2: \$0.1; I-3: \$4; and I-4: \$54). The nutrition intervention evaluated by the second study (47) was also more costly and more effective than usual care at a cost of $\$ 11$ per $1 \%$ cholesterol level reduction.

\section{Worksite physical activity and nutrition programs}

Six studies evaluated the cost-effectiveness of WHP programs aimed at increasing physical activity and improving nutrition $(48-50,52-54,57,58)$. Three of them $(48,49,52,54)$ only included intervention costs in their cost estimates. When costs were considered from a broader perspective $(50,53,57,58)$, intervention costs were partially offset by a reduction in absenteeism and/ or medical costs (table 3 ).

Three studies $(50,54,57,58)$ evaluated the costeffectiveness in terms of body weight reduction from various perspectives. All interventions were more costly and more effective than usual care. When only intervention costs were considered, the additional costs per kilogram body weight loss were $\$ 26$. When analyses were performed from the employer's perspective those costs were $\$ 75$ and $\$ 1534$, and from the societal perspective $\$ 174, \$ 20$, and $\$ 1282$ (see also table 4). One of those interventions (54) was also more costly and more effective in reducing waist circumference.

Two other studies $(48,49,52)$ evaluated the costeffectiveness by comparing intervention costs to the effect on CVD risk reduction. Both studies, however, used different composite scores to estimate the level of CVD risk reduction, which limits their comparability. The first study (52) evaluated three different interventions: (i) I-RFE: risk factor education, (ii) I-BC: behavioral counseling, and (iii) I-BCI: behavioral counseling plus incentives. All interventions were more costly and more effective than usual care (ICER I-RFE: \$10, I-BC: \$24, and I-BCI: \$363 per CVD risk unit reduced). The other study presented its results in two articles $(48,49)$, which differed in the number of CVD risk factors included in the composite score (ie, three risk factors in the first article versus four in the second article) as well as their control condition. Furthermore, in the first article (48), they did not include all intervention costs in their cost estimates (ie, fitness centre costs were missing). In the second article (49), they evaluated three different interventions: (i) I-FC: fitness centre, (ii) I-HEC: health education \& follow-up counseling, and (iii) I-HECE: health education, follow-up counseling \& environmental strategies. I-FC was more costly and less effective than usual care. The other interventions were more costly and more effective (ICER I-HEC: $\$ 2$ and \$2 I-HECE: $\$ 3$ and $\$ 3$ per 1\% of CVD risks, respectively highly or moderately reduced).

Another study (53) evaluated the cost-effectiveness from the employer's perspective using its effect on physical activity-related outcome measures. The intervention was more costly and more effective than usual care in increasing energy expenditure, and decreasing sub-maximal heart rate. However, the intervention was more costly and less effective in increasing the number of participants meeting physical activity recommendations.

\section{Cost-utility analysis}

One study (58) evaluated the cost-utility of both an internet- and a phone-based nutrition and physical 
Table 3. Costs (expressed in USD 2010 and rounded to the nearest dollar), outcomes, and incremental cost-effectiveness ratios of included

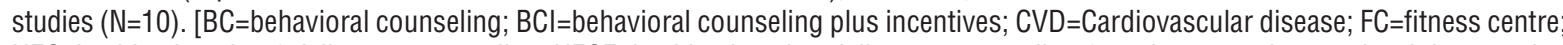
HEC=health education \& follow-up counseling; HECE=health education, follow-up counseling \& environmental strategies; I=Intervention group; NS=Not stated; PA=Physical activity; QALY=Quality-adjusted life year; RFE=risk factor education; SF-36=Short Form-36; UC=Usual care; WEL=Weight efficacy lifestyle; WPAI=Work productivity and activity impairment.]

\begin{tabular}{|c|c|c|c|}
\hline \multirow[t]{2}{*}{ Study } & Costs & Outcomes & \multirow{2}{*}{$\begin{array}{l}\text { Incremental cost-effectiveness } \\
\text { ratio }\end{array}$} \\
\hline & $\begin{array}{c}\text { Mean } \\
\text { [incremental] }\end{array}$ & Mean [incremental] & \\
\hline \multicolumn{4}{|c|}{ Worksite nutrition programs } \\
\hline \multirow[t]{5}{*}{ Katcher et al (51) } & \multirow[t]{5}{*}{$\begin{array}{l}\text { UC: } 0 \\
\text { I: } 226[226]\end{array}$} & $\begin{array}{l}\text { Body weight reduction }(\mathrm{kg}) \text { : } \\
\text { UC: }-0.1 ; 1: 5.1[5.3]^{\text {a }}\end{array}$ & 43 per kg body weight loss ${ }^{b}$ \\
\hline & & $\begin{array}{l}\text { Eating behavior (points) } \\
\text { Restraint: UC:-1.1; I: } 0.5[1.6]^{\mathrm{a}} \\
\text { Disinhibition: UC: } 0.1 ; 1: 1.7[1.7]^{\mathrm{a}} \\
\text { Hunger: UC: }-0.7 ; 1: 1.6[2.3]^{\mathrm{a}}\end{array}$ & $\begin{array}{l}\text { Costs per point decrease on the Eating Inventory subscales }{ }^{\text {b }} \\
\text { Restraint: }-141 \\
\text { Disinhibition: }-133 \\
\text { Hunger: }-98\end{array}$ \\
\hline & & $\begin{array}{l}\text { Health related quality of life (points) } \\
\text { Physical functioning: UC: } 0.23 ; \text { I: } 9.1 \text { [8.9 }^{\mathrm{a}} \\
\text { General health: UC: } 2.3 ; \text { I: } 12.6[10.3]^{\mathrm{a}} \\
\text { Physical role limitations: UC: }-2.3 ; \text { I: } 8.5[10.7] \\
\text { Emotional role limitations: UC: } 6.8 ; \text { I: } 8.7[1.9] \\
\text { Bodily pain: UC: -0.3; I: } 6.4 \text { [6.7] } \\
\text { Vitality: UC: -0.3; I: } 10.8[11]^{\mathrm{a}} \\
\text { Social functioning: UC: } 1.7 ; \text { I: } 6.2[4.4] \\
\text { Mental health: UC: }-1.5 ; \text { I: } 5.1[6.6]^{\mathrm{a}}\end{array}$ & $\begin{array}{l}\text { Costs per point improvement on the SF- } 36 \text { subscales }{ }^{\text {b }} \\
\text { Physical functioning: } 25 \\
\text { General health: } 22 \\
\text { Physical role limitations: } 21 \\
\text { Emotional role limitations: } 119 \\
\text { Bodily pain: } 34 \\
\text { Vitality: } 21 \\
\text { Social functioning: } 51 \\
\text { Mental health: } 34\end{array}$ \\
\hline & & $\begin{array}{l}\text { Work productivity (points) } \\
\text { Impairment at work: UC: } 2.0 ; \text { I: }-6.1 \text { [-8.1 }]^{\text {a }} \\
\text { Impairment with daily activities: UC: } 0.0 ; \text { I: }-9.8 \\
{[-9.8]^{\text {a }}}\end{array}$ & $\begin{array}{l}\text { Costs per point decrease on WPAI questionnaire subscales }{ }^{b} \\
\text { Impairment at work: } 28 \\
\text { Impairment with daily activities: } 23\end{array}$ \\
\hline & & Overall work impairment: UC: 1.4 . I: -6.2 [-7.6] & Overall work impairment: 30 \\
\hline \multirow[t]{4}{*}{ Siggaard et al (55) } & \multirow{4}{*}{$\begin{array}{l}\text { UC: NS } \\
\text { I: NS [NS] }\end{array}$} & Body weight reduction $(\mathrm{kg})$ : UC: $0.8 ;$ I: $4.2[3.4]^{\mathrm{a}}$ & 20 per kg body weight loss \\
\hline & & Reduction in overweight (\%): UC: $0.9 ; 1: 5[4.1]^{\text {a }}$ & 17 per $1 \%$ reduction in overweight \\
\hline & & $\begin{array}{l}\text { Daily carbohydrate intake (g): UC: } 3.1 \text {; I: } 36.0 \\
{[32.9]^{\text {a }}}\end{array}$ & 2 per gram increase in daily carbohydrate intake \\
\hline & & Daily fat intake (g): UC: -4.0 ; I: $-27.5[-23.5]^{\text {a }}$ & 3 per gram decrease in daily fat intake \\
\hline Wilson et al (56) & $\begin{array}{l}\text { UC: } 140 \\
\text { I-1: } 217[77] \\
\text { I-2: } 141[1] \\
\text { I-3: } 204[64] \\
\text { I-4: } 461[321]\end{array}$ & $\begin{array}{l}\text { Participants with a cholesterol level reduction of } \\
\geq 10 \%(\%): \text { UC: } 18.5 \\
\text { I-1: } 17.8[-0.7] \\
\text { I-2: } 28[9.5] \\
\text { I-3: } 33 \text { [14.5]; } \\
\text { I-4: } 24.5[6]\end{array}$ & $\begin{array}{l}\text { Costs per } 1 \% \text { of participants with a cholesterol level reduc- } \\
\text { tion of } \geq 10 \% \text { b } \\
\text { I-1: }-110 \\
\text { I-2: } 0.1 \\
\text { I-3: } 4 \\
\text { I-4: } 54\end{array}$ \\
\hline Byers et al (47) & $\begin{array}{l}\text { UC: } 33 \\
\text { I: } 72[39]\end{array}$ & $\begin{array}{l}\text { Levels of significance unknown } \\
\text { Cholesterol level reduction (\%) } \\
6 \text { months: UC: } 0.4 ; \text { I: } 1.2[0.8] \\
12 \text { months: UC: } 3 ; 1: 6.5 \%[3.5]^{\text {a }}\end{array}$ & $\begin{array}{l}6 \text { months: } 48 \text { per } 1 \% \text { cholesterol level reduction }{ }^{b} \\
12 \text { months: } 11 \text { per } 1 \% \text { cholesterol level reduction }{ }^{b}\end{array}$ \\
\hline \multicolumn{4}{|c|}{$\begin{array}{l}\text { Worksite physical activity and } \\
\text { nutrition programs }\end{array}$} \\
\hline \multirow[t]{5}{*}{ Rasu et al (54) } & \multirow{5}{*}{$\begin{array}{l}\text { UC: NS } \\
\text { I: NS [49] }\end{array}$} & Body weight reduction (kg): UC: -0.6 ; I: $1.3[1.9]^{\text {a }}$ & 26 per kg body weight loss \\
\hline & & $\begin{array}{l}\text { Waist circumference reduction (cm): UC: } 0.4 \\
\mathrm{I}: 2.1[1.7]^{\mathrm{a}}\end{array}$ & 29 per $\mathrm{cm}$ waist circumference reduction \\
\hline & & $\begin{array}{l}\text { Participants with a weight reduction of } \\
\geq 5 \% \text { (\%): UC: } 6.8 ; \text { I: } 22.6[15.8]^{\text {a }}\end{array}$ & 3 per $1 \%$ of participants with a weight reduction of $\geq 5 \%$ \\
\hline & & $\begin{array}{l}\text { Weight efficiency lifestyle (points): } \\
\text { Social pressure: UC: } 1.3 ; \text { I: } 2.5[1.3] \\
\text { Positive activity: UC: } 0.8 ; \text {;: } 2.3 \text { [1.5] } \\
\text { Availability: UC: } 2.2 ; \text { I: } 3.2 \text { [1.0] }\end{array}$ & \multirow[t]{2}{*}{$\begin{array}{l}\text { Costs per point improvement on the WEL questionnaire } \\
\text { subscales } \\
\text { Social pressure: } 38 \\
\text { Positive activity: } 33 \\
\text { Availability: } 49\end{array}$} \\
\hline & & Levels of significance unknown & \\
\hline $\begin{array}{l}\text { Groeneveld et al } \\
(50)\end{array}$ & $\begin{array}{l}\text { UC: } 5048 \\
\text { I: } 5399[351]\end{array}$ & Body weight reduction (kg): UC: $-1.1 ; \mathrm{l}: 1$ [2] a & 174 per $\mathrm{kg}$ body weight loss from the societal perspective \\
\hline \multirow[t]{2}{*}{$\begin{array}{l}\text { van Wier et al } \\
\text { (58) }\end{array}$} & \multirow{2}{*}{$\begin{array}{l}\text { UC: } 3150 \\
\text { I-1: } 3597 \\
{[447]} \\
\text { I-2: } 3168[18]\end{array}$} & $\begin{array}{l}\text { Body weight reduction (kg): UC: } 1.1 \text {; } \\
\text { I-phone: } 1.5[0.3] \text {; } \\
\text { I-Internet: } 1.9[0.9]\end{array}$ & $\begin{array}{l}\text { Costs per kg body weight loss from the societal perspective } \\
\text { I-phone: } 1282 \\
\text { I-Internet: } 20\end{array}$ \\
\hline & & $\begin{array}{l}\text { QALY: UC: } 1.85 \\
\text { I-phone: } 1.85[0.001] \\
\text { I-Internet: } 1.86[0.01]\end{array}$ & $\begin{array}{l}\text { Costs per QALY gained from the societal perspective } \\
\text { I-phone: } 311523 \\
\text { I-Internet: } 1698\end{array}$ \\
\hline
\end{tabular}


Table 3. Continued

\begin{tabular}{|c|c|c|c|}
\hline \multirow[t]{2}{*}{ Study } & Costs & Outcomes & \multirow{2}{*}{$\begin{array}{l}\text { Incremental cost-effectiveness } \\
\text { ratio }\end{array}$} \\
\hline & $\begin{array}{c}\text { Mean } \\
\text { [incremental] }\end{array}$ & Mean [incremental] & \\
\hline $\begin{array}{l}\text { Gussenhoven et } \\
\text { al (57) }\end{array}$ & $\begin{array}{l}\text { UC: } 4100 \\
\text { I-1: } 4469 \\
{[369]} \\
\text { I-2: } 4161[61]\end{array}$ & $\begin{array}{l}\text { Body weight reduction (kg) UC: } 1.6 \\
\text { I-phone: } 1.9[0.2] \\
\text { I-Internet: } 2.4[0.8]\end{array}$ & $\begin{array}{l}\text { Costs per kg body weight loss from the employer's } \\
\text { perspective } \\
\text { I-phone: } 1534 \\
\text { I-Internet: } 75\end{array}$ \\
\hline \multirow{3}{*}{$\begin{array}{l}\text { Oldenburg } \\
\text { et al (52) }\end{array}$} & \multirow{3}{*}{$\begin{array}{l}\text { UC: } 111 \\
\text { I-RFE: } 146 \\
\text { [35] } \\
\text { I-BC: } 274 \\
\text { [163] } \\
\text { I-BCl: } 285 \\
\text { [174] }\end{array}$} & CVD risk unit reduction & Costs per CVD risk unit reduced \\
\hline & & $\begin{array}{l}6 \text { months: UC: } 1.04 \\
\text { I-RFE: } 3.99[2.96]^{\mathrm{a}} \\
\text { I-BC: } 8.13[7.09]^{\mathrm{a}} \\
\text { I-BCI: } 4.16[4.01]^{\mathrm{a}}\end{array}$ & $\begin{array}{l}6 \text { months: } \\
\text { I-RFE: } 12 \\
\text { I-BC: } 23 \\
\text { I-BCI: } 43\end{array}$ \\
\hline & & $\begin{array}{l}12 \text { months: UC: }-0.76 \\
\text { I-RFE: } 2.79[3.55] \\
\text { I-BC: } 6.10[6.86]^{\text {a }} \\
\text { I-BCI: }-0.28[0.48]\end{array}$ & $\begin{array}{l}12 \text { months: } \\
\text { I-RFE: } 10^{\mathrm{b}} \\
\text { I-BC: } 24 \\
\text { I-BCI: } 363^{\text {b }}\end{array}$ \\
\hline \multirow[t]{4}{*}{ Erfurt et al (48) } & \multirow{4}{*}{$\begin{array}{l}\text { UC: } 5 \\
\text { I-HE: } 28 \text { [23] } \\
\text { I-HEC: } 50 \text { [45] } \\
\text { I-HECE: } 61 \\
\text { [56] }\end{array}$} & CVD risk reduction (\%) & Costs per $1 \%$ of 3 CVD risk factors reduced or prevented \\
\hline & & $\begin{array}{l}\text { High level reduction: UC: } 34 \\
\text { I-HE: } 35[1] \\
\text { I-HEC: } 44[10] \\
\text { I-HECE: } 46[12]\end{array}$ & $\begin{array}{l}\text { High level reduction: } \\
\text { I-HE: } 23 \\
\text { I-HEC: } 5 \\
\text { I-HECE: } 4\end{array}$ \\
\hline & & $\begin{array}{l}\text { Moderate level reduction: UC: } 40 \\
\text { I-HE: } 41[1] \\
\text { I-HEC: } 51[11] \\
\text { I-HECE: } 56[16]\end{array}$ & $\begin{array}{l}\text { Moderate level reduction: } \\
\text { I-HE: } 23 \\
\text { I-HEC: } 4 \\
\text { I-HECE: } 4\end{array}$ \\
\hline & & Levels of significance unknown & \\
\hline \multirow[t]{4}{*}{ Erfurt et al (49) } & \multirow{4}{*}{$\begin{array}{l}\text { UC: } 27 \\
\text { I-FC: } 61 \text { [33] } \\
\text { I-HEC: } 48 \text { [21] } \\
\text { I-HECE: } 60 \\
\text { [33] }\end{array}$} & CVD risk reduction (\%) & Costs per $1 \%$ of $4 \mathrm{CVD}$ risk factors that reduced or prevented \\
\hline & & $\begin{array}{l}\text { High level reduction: UC: } 35 \\
\text { I-FC } 32 \text { [-3]; } \\
\text { I-HEC: } 44 \text { [9]; } \\
\text { I-HECE: } 45 \text { [10] }\end{array}$ & $\begin{array}{l}\text { High level reduction: } \\
\text { I-FC: }-11 \\
\text { I-HEC: } 2 \\
\text { I-HECE: } 3\end{array}$ \\
\hline & & $\begin{array}{l}\text { Moderate level reduction: UC: } 39 \text {; } \\
\text { I-FC: } 36 \text { [-3]; } \\
\text { I-HEC: } 48 \text { [9]; } \\
\text { I-HECE: } 51 \text { [12] }\end{array}$ & $\begin{array}{l}\text { Moderate level reduction: } \\
\text { I-FC: }-11 \\
\text { I-HEC: } 2 \\
\text { I-HECE: } 3\end{array}$ \\
\hline & & Levels of significance unknown & \\
\hline \multirow[t]{4}{*}{ Proper et al (53) } & \multirow[t]{4}{*}{$\begin{array}{l}\text { UC: } 2591^{\mathrm{c}} \\
\text { I: } 2979[387]^{\mathrm{c}}\end{array}$} & $\begin{array}{l}\text { Increase in participants meeting PA } \\
\text { recommendations (\%): UC: }-6 ; \text { I: }-6.6[-0.6]\end{array}$ & $\begin{array}{l}-1308 \text { per } 1 \% \text { increase in participants meeting PA } \\
\text { recommendations from the employer's perspective }\end{array}$ \\
\hline & & $\begin{array}{l}\text { Increase in energy expenditure } \\
\text { (kilocalorie/day): UC: }-129 ; \text { I: } 64.2 \text { [193.2] }{ }^{\text {a }}\end{array}$ & 7 per extra kilocalorie/day from the employer's perspective \\
\hline & & $\begin{array}{l}\text { Decrease in sub-maximal heart rate } \\
\text { (beats/minute): UC: }-2.5 ; 1: 2.2[4.7]^{\text {a }}\end{array}$ & $\begin{array}{l}299 \text { per beat/minute decrease in sub-maximal heart rate from } \\
\text { the employer's perspective }\end{array}$ \\
\hline & & $\begin{array}{l}\text { Decrease in participants with upper-extremity } \\
\text { symptoms (\%): UC: } 6.2 ; \mathrm{I}: 17.9[-11.7]\end{array}$ & $\begin{array}{l}68 \text { per } 1 \% \text { decrease in participants with upper-extremity } \\
\text { symptoms from the employer's perspective }\end{array}$ \\
\hline
\end{tabular}

a Significant at $\mathrm{P}<0.05$.

${ }^{b}$ Incremental cost-effectiveness ratio calculated based on the information provided in the article and other related materials.

c Average costs of all participants with complete cost and effect data. Costs included in the cost-effectiveness analyses were variable and depended on the number of participants with complete follow-up data in terms of that outcome measure. 
activity program. Analyses were conducted from the societal perspective. After 24 months, the cost-utility of the internet-based intervention was $\$ 1698$ per qualityadjusted life year (QALY) gained and that of the phonebased intervention \$311523 per QALY gained.

\section{Discussion}

The present review critically appraised and summarized the current evidence on the cost-effectiveness of worksite physical activity and/or nutrition programs. Ten studies (published in 12 articles), evaluating 18 programs, were included in the review.

None of the studies evaluated WHP programs aimed solely at increasing physical activity.

From various perspectives, worksite nutrition as well as worksite physical activity and nutrition programs $(\mathrm{N}=6)$ were more costly and more effective in reducing body weight compared to usual care during the first years after implementation. If only intervention costs were considered, most worksite nutrition $(\mathrm{N}=4 / 5)$ and worksite physical activity and nutrition programs $(\mathrm{N}=5 / 6)$ were more costly and more effective in reducing cholesterol level and CVD risks, respectively. Currently, however, there are no set levels for how much different stakeholders are willing to pay for reductions in body weight, cholesterol level, and CVD risks. It is therefore unknown whether the costs associated with achieving these results are acceptable, ie, whether these programs are cost-effective. Therefore, it is up to individual decision-makers to judge whether or not these programs offer value for money.
CEA were also conducted in terms of various other outcome measures (eg, dietary habits, quality of life, physical activity-related outcome measures, and workrelated outcome measures). However, ICER in terms of these outcome measures were only calculated for one intervention. Furthermore, only one study evaluated the cost-utility of worksite physical activity and nutrition programs and provided mixed results. When compared to the National Institute for Health and Clinical Excellence (NICE) threshold of GBP20,000 ( \pm USD30 500) to GBP30 000 ( \pm USD45 700) per QALY gained as well as the frequently cited US threshold of USD50 000-100 000 per QALY gained (59), the internet-based intervention of the study can be regarded as cost-effective (\$1698 per QALY gained), whereas the phone-based intervention (\$311 523 per QALY gained) cannot. All in all, these findings do not necessarily support the conclusion of a previous review (36) that the literature provides "guarded cautious optimism" about the cost-effectiveness of WHP programs.

When only intervention costs were considered, the additional costs per kilogram body weight loss ranged from \$20-43, independent of the program focus (ie, nutrition or physical activity and nutrition). From a broader perspective, intervention costs were partially offset by a reduction in medical and/or absenteeism costs. Strikingly, this did not result in lower ICER as the three programs evaluated by only including intervention costs were equally or more effective in reducing body weight compared to those evaluated from a broader perspective, whereas their intervention costs were similar or lower. This superior effectiveness might be explained by the fact that these studies conducted follow-up measurements immediately after the intervention period $(<6$

Table 4. Main characteristics and outcomes of studies evaluating both costs and reductions in body weight. Costs are expressed in USD 2010 and rounded to the nearest dollar [PA=Physical activity; NS=Not stated]

\begin{tabular}{|c|c|c|c|c|c|c|c|}
\hline Study & $\begin{array}{l}\text { Focus of } \\
\text { interven- } \\
\text { tion }\end{array}$ & $\begin{array}{l}\text { Intervention } \\
\text { duration }\end{array}$ & $\begin{array}{l}\text { Follow-up } \\
\text { duration }\end{array}$ & $\begin{array}{l}\text { Incremental } \\
\text { intervention } \\
\text { costs (per } \\
\text { participant) }\end{array}$ & $\begin{array}{l}\text { Incremental } \\
\text { total costs (per } \\
\text { participant) }\end{array}$ & $\begin{array}{l}\text { Weight } \\
\text { reduction } \\
\text { (kilograms) }\end{array}$ & $\begin{array}{l}\text { Incremental } \\
\text { cost- } \\
\text { effectiveness } \\
\text { ratio }\end{array}$ \\
\hline \multicolumn{8}{|l|}{$\begin{array}{l}\text { Only considering } \\
\text { intervention costs }\end{array}$} \\
\hline Katcher et al (51) & Diet & 22 weeks & 22 weeks & 226 & 226 & $5.3^{a}$ & $43^{b}$ \\
\hline Siggaard et al (55) & Diet & 12 weeks & 12 weeks & NS & NS & $3.4^{\mathrm{a}}$ & 20 \\
\hline Rasu et al (54) & PA \& diet & 6 months & 6 months & 49 & 49 & $1.9^{a}$ & 26 \\
\hline $\begin{array}{l}\text { Employer's perspective } \\
\text { Gussenhoven et } \\
\text { al (57) }\end{array}$ & PA \& diet & 6 months & 12 months & $\begin{array}{c}256 \text { (I-Phone) } \\
227 \text { (I-Internet) }\end{array}$ & $\begin{array}{l}369 \text { (I-Phone) } \\
61 \text { (I-Internet) }\end{array}$ & $\begin{array}{c}0.2 \text { (I-Phone) } \\
0.8 \text { (I-Internet) }\end{array}$ & $\begin{array}{l}1534 \text { (I-Phone) } \\
75 \text { (I-Internet) }\end{array}$ \\
\hline \multicolumn{8}{|l|}{ Societal perspective } \\
\hline van Wier et al (58) & PA \& diet & 6 months & 24 months & $\begin{array}{c}256 \text { (I-Phone) } \\
227 \text { (I-Internet) }\end{array}$ & $\begin{array}{l}447 \text { (I-Phone) } \\
18 \text { (I-Internet) }\end{array}$ & $\begin{array}{c}0.3 \text { (I-Phone) } \\
0.9 \text { (I-Internet) }\end{array}$ & $\begin{array}{l}1282 \text { (I-Phone) } \\
20 \text { (I-Internet) }\end{array}$ \\
\hline Groeneveld et al (50) & PA \& diet & 6 months & 12 months & 730 & 351 & $2.0^{a}$ & 174 \\
\hline
\end{tabular}

a Significant at $P<0.05$.

${ }^{\mathrm{b}}$ Incremental cost-effectiveness ratio calculated based on the information provided in the article and related materials. 
months) as opposed to several months after the completion of the program ( $\geq 6$ months) in the studies performed from a broader perspective. Systematic reviews show that (partial) weight rebound after the intervention period is common $(60,61)$. Another explanation may be the non-randomized design of two of these three studies (ie, results may be confounded by selection bias) (40). Nevertheless, it would also be insightful to investigate the relationship between intervention costs, which are strongly related to intervention composition and intensity, and effect size in more detail. If it is established that more costly programs do not necessarily produce better health outcomes or cost-savings, cost containment strategies during the design phase of a program may be a useful strategy to optimize cost-effectiveness.

A risk of bias assessment revealed that most of the included studies had several methodological shortcomings. For example, few studies specifically stated their perspective and an incremental analysis of costs and effects were not performed in all studies. Furthermore, many studies applied a rather restrictive perspective by only including intervention costs in their cost estimate. However, as WHP programs are thought to be associated with other cost categories (eg, medical and productivityrelated costs) (27), the adoption of a broader perspective is recommended. Costs were only measured in physical units in four studies, and of these, only one valued them appropriately by calculating them based on depleted sources and stating their reference year.

Furthermore, although research has indicated that presenteeism accounts for a larger proportion of productivity-related losses compared to absenteeism, none of the studies conducted from the societal and/or employer's perspective included presenteeism costs in their cost estimates. This likely resulted from the fact that a "gold standard" for measuring and valuing presenteeism does not exist. Nevertheless, up until now, various instruments have been developed to measure presenteeism, of which several capture lost productivity suitable for direct translation into a monetary unit (62-64).

In addition, although economic analyses require that assumptions are made $(30,65)$, few studies conducted a sensitivity analysis and hardly any of the studies reported on the uncertainty around their ICER. Sensitivity analyses are useful to test the robustness of the study results, but do not give insight into the uncertainty due to sampling variation $(30,66,67)$. To quantify precision, non-parametric bootstrapping can be used as a statistical technique for dealing with the highly skewed nature of cost data $(30,65)$ and the uncertainty around an ICER can be illustrated graphically using cost-effectiveness planes (30). It is also important to mention that three studies did not even report on the uncertainty around their effect sizes. Economic evaluations rely heavily on the assessment of the clinical effectiveness (30).
Not reporting on the uncertainty around the effect sizes strongly hampers the interpretation of the reported ICER. Using results of economic evaluations with a high risk of bias for deciding how resources should be optimally allocated, may lead to inappropriate decisions $(40,65)$. Therefore, strong conclusion about the costeffectiveness of worksite physical activity and/or nutrition programs cannot be made due to the methodological shortcomings of the included studies. This should be addressed in future studies. In particular, future studies should include presenteeism costs and emphasis should be placed on the handling of uncertainty.

One of the main strengths of this review was that it incorporated a risk of bias assessment using a standardized quality checklist based on consensus among experts in the field of economic evaluations. Furthermore, four additional studies were identified compared to previous reviews on the cost-effectiveness of WHP programs, all of which evaluated costs and kilogram body weight loss. As a result, the present review was the first to compare ICER in terms of costs per kilogram body weight loss from different perspectives. However, due to heterogeneity of outcome measures, follow-up (long- versus short-term), and perspectives, results could not be pooled. As a result of the relatively limited number of included studies, it was also not possible to conduct subgroup analyses to investigate the impact of program format [ie, (self-)assessment, educational/ informational, behavioral, exercise, environmental, and incentive components] or participant characteristics (eg, age, gender, and white- versus blue-collar workers) on the cost-effectiveness of the interventions.

Therefore, the present review cannot indicate which program formats are important for attaining cost-effectiveness or how worksite physical activity and/or nutrition programs should optimally be designed. Furthermore, a program's cost-effectiveness may depend on the characteristics of its participants. Blue-collar workers, for example, may respond differently compared to white-collar workers as a result of their difference in underlying health risks (68). It is important to address these issues in future reviews when additional research on the cost-effectiveness of worksite physical activity and/or nutrition programs has been completed.

Another limitation of the present review was the possible effect of publication bias. That is, economic evaluations may be more likely to be conducted of interventions that had previously been found to be effective, and studies with favorable results may be more likely to be published. It is also important to bear in mind that all CEA conducted from the employer's perspective were performed in The Netherlands. These results are not necessarily generalizable to other countries, as their health and social security systems may differ. US employers, for example, bear 
a large part of the medical costs of their employees, whereas in Europe these accrue to the government and/or insurance companies (28). Furthermore, only trial-based economic evaluations with relatively short follow-ups ( $\leq 3$ years) were identified and included. As cost-savings due to improved health might only occur after a longer period, this may have resulted in an underestimation of a possible absenteeism and/or medical cost-offset effect. Due to their relatively short follow-ups, studies were also only able to assess the programs' cost-effectiveness in terms of intermediate outcome measures relating to aspects of diet and physical activity (eg, CVD risk, body weight, and cholesterol level reduction), whereas disease prevention (eg, CVD, diabetes type 2) can be regarded as the primary endpoint of worksite physical activity and/or nutrition programs (69). To bridge the gap between what has been observed in the trial-based economic evaluations and what the cost-effectiveness of worksite physical activity and/or nutrition programs would be over a longer time horizon, decision analytic modeling could be used (30). However, currently little is known about the longevity of the intermediate outcomes of WHP programs and the relationship of these outcomes with changes in long-term medical and productivity-related costs. More research should be done in this field to allow for the development of credible decision analytic models.

\section{Concluding remarks}

Current evidence indicates that worksite physical activity and/or nutrition programs can result in reductions in body weight, cholesterol level, and CVD risks, but at a higher cost than usual care. Because it is unknown how much decision-makers are willing to pay for these health outcomes, conclusions about their cost-effectiveness cannot be made. Most of the included studies had several methodological shortcomings, which hinders the validity of their results. Therefore, there is substantial need for improvement of the methodological quality of studies evaluating the cost-effectiveness of worksite physical activity and/or nutrition programs and particular emphasis should be placed on the handling of uncertainty.

\section{Acknowledgements}

We thank Marijke Mol for her contribution in developing the search strategy.

\section{References}

1. Hankinson AL, Daviglus ML, Bouchard C, Carnethon M, Lewis CE, Schreiner PJ, et al. Maintaining a high physical activity level over 20 years and weight gain. JAMA. 2010;304(23):2603-10. http://dx.doi.org/10.1001/ jama.2010.1843.

2. Jakicic JM, Otto AD. Physical activity considerations for the treatment and prevention of obesity. Am J Clin Nutr. 2005;82(1):226S-9S.

3. Kumanyika SK, Obarzanek E, Stettler N, Bell R, Field AE, Fortmann SP, et al. Population-based prevention of obesity. The need for comprehensive promotion of healthful eating, physical activity, and energy balance. A scientific statement from American heart association council on epidemiology and prevention, interdisciplinary committee for prevention (formerly the expert panel on population and prevention science). Circulation. 2008;118(4):428-64. http://dx.doi. org/10.1161/CIRCULATIONAHA.108.189702.

4. Mozaffarian D, Hao T, Rimm EB, Willett WC, Hu FB Changes in diet and lifestyle and long-term weight gain in women and men. N Engl J Med. 2011;364(25):2392-404. http://dx.doi.org/10.1056/NEJMoa1014296.

5. Diet, nutrition and the prevention of chronic diseases: Report of a Joint WHO/FAO Expert Consultation. Geneva: World health organization; 2003.

6. Bennett GG, Wolin KY, Puleo EM, Masse LC, Atienza AA. Awareness of national physical activity recommendations for health promotion among US adults. Med Sci Sports Exerc. 2009;41(10):1849-55.http://dx.doi.org/10.1249/ MSS.0b013e3181a52100.

7. Krebs-Smith SM, Cleveland LE, Ballard-Barbash R, Cook DA, Kahle LL. Characterizing food intake patterns of American adults. Am J Clin Nutr. 1997;65(4):1264S-8S.

8. Martínez-González MA, Varo JJ, Santos JL, De Irala J, Gibney M, Kearney J, et al. Prevalence of physical activity during leisure time in the European Union. Med Sci Sports Exerc. 2001;33(7):1142-6.

9. Pronk NP, Anderson LH, Crain AL, Martinson BC, O'Connor PJ, Sherwood NE, et al. Meeting recommendations for multiple healthy lifestyle factors: Prevalence, clustering, and predictors among adolescent, adult, and senior health plan members. Am J Prev Med. 2004;27(2, Supplement 1):25-33. http://dx.doi.org/10.1016/j.amepre.2004.04.022.

10. Troiano RP, Berrigan D, Dodd KW, Masse LC, Tilbert T, McDowell M. Physical activity in the United States measured by accelerometer. Med Sci Sports Exerc. 2008;40(1):181-8.

11. Troiano RP, Macera CA, Ballard-Barbash R. Be physically active each day. How can we know? J Nutr. 2001;131(2):451S-60S

12. Flegal KM, Carroll MD, Ogden CL, Johnson CL. Prevalence and trends in obesity among US adults, 1999-2000. JAMA. 2002;288(14):1723-7. http://dx.doi.org/10.1001/ jama.288.14.1723.

13. Adult overweight and obesity in the European Union (EU27) based on measured height and weight [Internet]. International 
organisation for the study of obesity. May 2011 [Cited May 2011]. Available from: http://www.iaso.org/site_media/ uploads/Adult_EU_27_May_2011.pdf.

14. Trogdon JG, Finkelstein EA, Hylands T, Dellea PS, KamalBahl SJ. Indirect costs of obesity: a review of the current literature. Obes Rev. 2008;9(5):489-500. http://dx.doi. org/10.1111/j.1467-789X.2008.00472.x.

15. Yach D, Stuckler D, Brownell KD. Epidemiologic and economic consequences of the global epidemics of obesity and diabetes. Nat Med. 2006;12(1):62-6. http://dx.doi. org/10.1038/nm0106-62.

16. Thompson D, Edelsberg J, Colditz GA, Bird AP, Oster G. Lifetime health and economic consequences of obesity. Arch Intern Med. 1999;159(18):2177-83. http://dx.doi. org/10.1001/archinte.159.18.2177.

17. Goetzel RZ, Ozminkowski RJ. The health and cost benefits of work site health-promotion programs. Annu Rev Public Health. 2008;29:303-23. http://dx.doi.org/10.1146/annurev. publhealth.29.020907.090930.

18. National Institute for Health and Clinical Excellence (NICE). Workplace health promotion: How to encourage employees to be physically active. London: NICE; 2008. NICE Public Health Guidance 13.

19. NiMhurchu C, Aston L, Jebb S. Effects of worksite health promotion interventions on employee diets: a systematic review. BMC Public Health. 2010;10:62. http://dx.doi. org/10.1186/1471-2458-10-62.

20. Anderson LM, Quinn TA, Glanz K, Ramirez G, Kahwati LC, Johnson DB, et al. The effectiveness of worksite nutrition and physical activity interventions for controlling employee overweight and obesity: A systematic review. Am J Prev Med. 2009;37(4):340-57. http://dx.doi.org/10.1016/j. amepre.2009.07.003.

21. Groeneveld IF, Proper KI, van der Beek AJ, Hildebrandt $\mathrm{VH}$, van Mechelen W. Lifestyle-focused interventions at the workplace to reduce the risk of cardiovascular disease-a systematic review. Scand J Work Environ Health. 2010;36(3):202-15. http://dx.doi.org/10.5271/sjweh.2891.

22. Verweij LM, Coffeng J, van Mechelen W, Proper KI. Metaanalyses of workplace physical activity and dietary behaviour interventions on weight outcomes. Obes Rev. 2011;(12):40629. http://dx.doi.org/10.1111/j.1467-789X.2010.00765.x.

23. Weinstein MC, Stason WB. Foundations of cost-effectiveness analysis for health and medical practices. N Engl J Med. 1977;296(13):716-21. http://dx.doi.org/10.1056/ NEJM197703312961304.

24. Burdorf A. Economic evaluation in occupational health--its goals, challenges, and opportunities. Scand J Work Environ Health. 2007;33(3):161-4. http://dx.doi.org/10.5271/ sjweh. 1127.

25. Leigh JP. Expanding research on the economics of occupational health. Scand J Work Environ Health. 2006;32(1):1-4. http:// dx.doi.org/10.5271/sjweh.969.

26. Miller P, Haslam C. Why employers spend money on employee health: Interviews with occupational health and safety professionals from British Industry. Safety Science. 2009;47(2):163-9. http://dx.doi.org/10.1016/j. ssci.2008.04.001.

27. Nicholson S, Pauly MV, Polsky D, Baase CM, Billotti GM, Ozminkowski RJ, et al. How to present the business case for healthcare quality to employers. Appl Health Econ Health Policy 2005;4(4):209-18.http://dx.doi. org/10.2165/00148365-200504040-00003.

28. van Dongen JM, Proper KI, van Wier MF, van der Beek AJ, Bongers PM, Van Mechelen W, et al. Systematic review on the financial return of worksite health promotion programmes aimed at improving nutrition and/or increasing physical activity. Obes Rev. 2011;12(12):1031-49. http://dx.doi. org/10.1111/j.1467-789X.2011.00925.x.

29. Oster G, Thompson D, Edelsberg J, Bird AP, Colditz GA. Lifetime health and economic benefits of weight loss among obese persons. Am J Public Health 1999;89(10):1536-42. http://dx.doi.org/10.2105/AJPH.89.10.1536.

30. Drummond MF, Sculpher MJ, Torrance GW, O'Brien BJ, Stoddart GL. Methods for the Economic Evaluation of Health Care Programmes. 3rd edition. New York: Oxford University Press; 2005.

31. Pelletier KR. A review and analysis of the health and costeffective outcome studies of comprehensive health promotion and disease prevention programs at the worksite: 1991-1993 update. Am J Health Promot. 1993;8(1):50-62. http://dx.doi. org/10.4278/0890-1171-8.1.50.

32. Pelletier KR. A review and analysis of the health and costeffective outcome studies of comprehensive health promotion and disease prevention programs at the worksite: 1993-1995 update. Am J Health Promot. 1996;10(5):380-8. http://dx.doi. org/10.4278/0890-1171-10.5.380.

33. Pelletier KR. Clinical and cost outcomes of multifactorial, cardiovascular risk management interventions in worksites: a comprehensive review and analysis. J Occup Environ Med. 1997;39(12):1154-69. http://dx.doi.org/10.1097/00043764199712000-00009.

34. Pelletier KR. A review and analysis of the clinical and costeffectiveness studies of comprehensive health promotion and disease management programs at the worksite: 1995-1998 update (IV). Am J Health Promot. 1999;13(6):333-45, iii. http://dx.doi.org/10.4278/0890-1171-13.6.333.

35. Pelletier KR. A review and analysis of the clinical- and costeffectiveness studies of comprehensive health promotion and disease management programs at the worksite: 1998-2000 update. Am J Health Promot. 2001;16(2):107-16. http:// dx.doi.org/10.4278/0890-1171-16.2.107.

36. Pelletier KR. A review and analysis of the clinical and costeffectiveness studies of comprehensive health promotion and disease management programs at the worksite: update VI 2000-2004. J Occup Environ Med. 2005;47(10):1051-8. http://dx.doi.org/10.1097/01.jom.0000174303.85442.bf.

37. Pelletier KR. A review and analysis of the clinical and costeffectiveness studies of comprehensive health promotion and disease management programs at the worksite: Update VII 2004-2008. J Occup Environ Med. 2009;51(7):822-37. http:// 
dx.doi.org/10.1097/JOM.0b013e3181a7de5a.

38. Proper KI, Van Mechelen W. Effectiveness and economic impact of worksite interventions to promote physical activity and healthy diet Background paper prepared for the WHO/ WEF Joint Event on Preventing Noncommunicable Diseases in the Workplace (Dalian/ China, September 2007). Geneva: WHO press; 2008.

39. Soler RE, Leeks KD, Razi S, Hopkins DP, Griffith M, Aten A, et al. A systematic review of selected interventions for worksite health promotion: The assessment of health risks with feedback. Am J Prev Med. 2010;38(2, Supplement 1):S237S262. http://dx.doi.org/10.1016/j.amepre.2009.10.030.

40. Higgins JPT, Green S (editors). Cochrane Handbook for Systematic Reviews of Interventions Version 5.1.0 [updated March 2011]. The Cochrane Collaboration, 2011. Available from: http://cochrane-handbook.org.

41. Jefferson T, Demicheli V, Vale L. Quality of systematic reviews of economic evaluations in health care. JAMA. 2002;287(21):2809-12. http://dx.doi.org/10.1001/ jama.287.21.2809.

42. Chapman LS. Meta-evaluation of worksite health promotion economic return studies: 2005 update. Am J Health Promot.2005;19(6):1-11.

43. Evers S, Goossens M, de Vet H, van Tulder M, Ament A. Criteria list for assessment of methodological quality of economic evaluations: Consensus on Health Economic Criteria. Int J Technol Assess Health Care. 2005;21(02):240-5.

44. Gerkens S, Crott R, Cleemput I, Thissen JP, Closon MC, Horsmans $\mathrm{Y}$, et al. Comparison of three instruments assessing the quality of economic evaluations: A practical exercise on economic evaluations of the surgical treatment of obesity. Int J Technol Assess Health Care. 2008;24(03):318-25. http:// dx.doi.org/10.1017/S0266462308080422.

45. U.S. Bureau of Labor Statistics [Internet]. [Cited 2010 December 1]. Available from: http://www.bls.gov.

46. Organisation for Economic Co-operation and Development (OECD). StatExtracts. OECD Selected Data [Internet]. [Cited December 1 2010]. Available from: http://stats.oecd.org/ Index.aspx.

47. Byers T, Mullis R, Anderson J, Dusenbury L, Gorsky R, Kimber C, et al. The costs and effects of a nutritional education program following work-site cholesterol screening. Am J Public Health. 1995;85(5):650-5. http://dx.doi.org/10.2105/ AJPH.85.5.650.

48. Erfurt JC, Foote A, Heirich MA. The cost-effectiveness of worksite wellness programs for hypertension control, weight loss, and smoking cessation. J Occup Med. 1991;33(9):962-70.

49. Erfurt J, FooteA, Heinrich M. The cost-effectiveness of worksite wellness programs for hypertension control, weight loss, smoking cessation, and exercise. Pers Psychol. 1992;45(1):527. http://dx.doi.org/10.1111/j.1744-6570.1992.tb00842.x.

50. Groeneveld IF, van Wier MF, Proper K, Bosmans JE, van Mechelen W, van der Beek A. Cost-effectiveness and cost-benefit of a lifestyle intervention for workers in the construction industry at risk for cardiovascular disease. J Occup Environ Med. 2011;53(6):610-17. http://dx.doi.
org/10.1097/JOM.0b013e31821b9c24.

51. Katcher HI, Ferdowsian HR, Hoover VJ, Cohen JL, Barnard ND. A Worksite vegan nutrition program is well-accepted and improves health-related quality of life and work productivity. Ann Nutr Metab. 2010;56(4):245-52. http://dx.doi. org/10.1159/000288281.

52. Oldenburg B, Owen N, Parle M, Gomel M. An economic evaluation of four work site based cardiovascular risk factor interventions. Health Educ Behav. 1995;22(1):9-19. http:// dx.doi.org/10.1177/109019819502200103.

53. Proper KI, de Bruyne MC, Hildebrandt VH, van der Beek AJ, Meerding WJ, van MW. Costs, benefits and effectiveness of worksite physical activity counseling from the employer's perspective. Scand J Work Environ Health. 2004;30(1):36-46. http://dx.doi.org/10.5271/sjweh.763.

54. Rasu RS, Hunter CM, Peterson AL, Maruska HM, Foreyt JP. Economic evaluation of an Internet-based weight management program. Am J Manag Care. 2010;16(4):e98-104.

55. Siggaard R, Raben A, Astrup A. Weight loss during 12 week's ad libitum carbohydrate-rich diet in overweight and normal-weight subjects at a Danish work site. Obes Res. 1996;4(4):347-56.

56. Wilson MG, Edmunson J, DeJoy DM. Cost Effectiveness of work-site cholesterol screening and intervention programs. J Occup Environ Med. 1992;34(6):642-9.

57. Gussenhoven AHM, van Wier MF, Bosmans JE, Dekkers JC, van Mechelen W. Economic evaluation of a distance counselling lifestyle programme among overweight employees from a company perspective, Alife@Work: a randomized controlled trial. Work. In press 2012

58. van Wier MF, Dekkers JC, Bosmans JE, Heymans MW, Hendriksen IJM, Pronk NP et al. Economic evaluation of a weight control program with e-mail and telephone counseling among overweight employees: a randomized controlled trial. Int J Behav Nutr Phys Act. In press 2012.

59. Ubel PA, Hirth RA, Chernew ME, Fendrick AM. What is the -price of life and why doesn't it increase at the rate of inflation? Arch Intern Med. 2003;163(14):1637-41. http://dx.doi. org/10.1001/archinte.163.14.1637.

60. Barte JCM, Ter Bogt NCW, Bogers RP, Teixeira PJ, Blissmer $\mathrm{B}$, Mori TA, et al. Maintenance of weight loss after lifestyle interventions for overweight and obesity, a systematic review. Obes Rev 2010;11(12):899-906. http://dx.doi.org/10.1111/ j.1467-789X.2010.00740.x.

61. Loveman E, Frampton GK, Shepherd J, Picot J, Cooper $\mathrm{K}$, Bryant $\mathrm{J}$, et al. The clinical effectiveness and costeffectiveness of long-term weight management schemes for adults: a systematic review. Health Technol Assess 2011;15(2):1-182.

62. Mattke S, Balakrishnan A, Bergamo G, Newberry SJ. A review of methods to measure health-related productivity loss. Am J Manag Care. 2007;13(4):211-7.

63. Koopmanschap MA. PRODISQ: a modular questionnaire on productivity and disease for economic evaluation studies. Expert Rev Pharmacoecon Outcomes Res. 2005;5(1):23-8. http://dx.doi.org/10.1586/14737167.5.1.23. 
64. Koopmanschap M, Meeding WJ, Evers S, Severens J, Burdorf A, Brouwer W. Handleiding voor het gebruik van PRODISQ versie 2.1. [Handbook on use of PRODISQ.] Rotterdam/ Maastricht, Erasmus MC - Instituut voor Medical Technology Assessment, Instituut Maatschappelijke Gezondheidszorg, Universiteit van Maastricht - Beleid Economie en Organisatie van de Zorg; 2004.

65. Uegaki K, de Bruijne MC, Lambeek L, Anema JR, van der Beek AJ, van MW, et al. Economic evaluations of occupational health interventions from a corporate perspective - a systematic review of methodological quality. Scand J Work Environ Health. 2010;36(4):273-88. http://dx.doi.org/10.5271/ sjweh.3017.

66. Briggs AH, Gray AM. Handling uncertainty when performing economic evaluation of healthcare interventions. Health Technol Assess. 1999;3(2):1-134.
67. Sendi P, Gafni A, Birch S. Opportunity costs and uncertainty in the economic evaluation of health care interventions. Health Econ. 2002;11:23-31. http://dx.doi.org/10.1002/hec.641.

68. Baicker K, Cutler D, Song Z. Workplace wellness programs can generate savings. Health Aff (Millwood). 2010;29(2):304 11. http://dx.doi.org/10.1377/hlthaff.2009.0626.

69. Prentice RL, Willett WC, Greenwald P, Alberts D, Bernstein L, Boyd NF, et al. Nutrition and physical activity and chronic disease prevention: research strategies and recommendations. J Natl Cancer Inst. 2004;96(17):1276-87. http://dx.doi. org/10.1093/jnci/djh240.

70. Levin SM, Ferdowsian HR, Hoover VJ, Green AA, Barnard ND. A worksite programme significantly alters nutrient intakes. Public Health Nutr. 2010;13(10):1629-35. http:// dx.doi.org/10.1017/S136898000999303X.

Received for publication: 19 October 2011

\section{Appendix}

\section{EMBASE search strategy}

\begin{tabular}{|c|c|}
\hline Combined search & (\#1 AND \#2 AND \#3 AND \#4) NOT \#5 \\
\hline \#1 Intervention & $\begin{array}{l}\text { 'health promotion'/exp OR ‘harm reduction'/exp OR 'high risk behavior'/exp OR ‘risk reduction'/exp OR 'health behav- } \\
\text { ior'/de OR 'primary prevention'/exp OR 'secondary prevention'/exp OR 'occupational health'/exp OR health:ab,ti OR } \\
\text { intervention:ab,ti OR 'life style':ab,ti OR lifestyle:ab,ti OR prevention:ab,ti OR preventive:ab,ti OR 'risk factor':ab,ti OR } \\
\text { 'risk factors':ab,ti NOT 'rehabilitation'/exp }\end{array}$ \\
\hline \#2 Intervention aim & 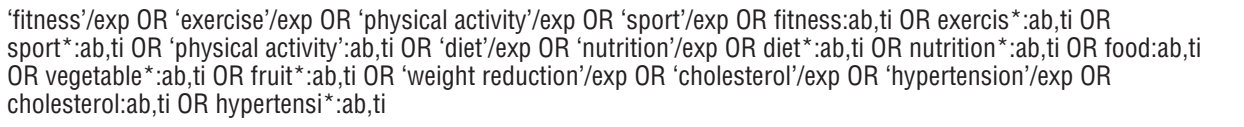 \\
\hline $\begin{array}{l}\text { \#3 Participants } \\
\text { (Population / Setting) }\end{array}$ & $\begin{array}{l}\text { 'manpower'/exp OR ‘workplace'/exp OR employ*:ab,ti OR worker*:ab,ti OR workplace*:ab,ti OR ‘work site’:ab,ti OR } \\
\text { personnel*:ab,ti OR workforce:ab,ti OR staff:ab,ti }\end{array}$ \\
\hline \#4 Study design & $\begin{array}{l}\text { ‘economic evaluation'/exp OR 'economic evaluation':ab,ti OR ‘economic analysis':ab,ti OR (cost:ab,ti OR costs:ab,ti AND } \\
\text { (benefit”:ab,ti OR utilit*:ab,ti OR effective*:ab,ti OR minimi?ation:ab,ti)) OR ROl:ab,ti OR “return on investment”:ab,ti }\end{array}$ \\
\hline \#5 Limits & ‘newborn'/exp OR ‘child'/exp OR 'adolescent'/exp NOT 'adult'/exp \\
\hline
\end{tabular}

